\title{
AVIFAUNA OF BHITARKANIKA MANGROVES, INDIA
}

\author{
G.V. Gopi ${ }^{1,2}$ and Bivash Pandav ${ }^{3,4}$
}

1,3 Wildlife Institute of India, Post Box 18, Chandrabani, Dehradun, Uttarakhand 248001, India

2 (presently) G.B. Pant Institute of Himalayan Environment and development, NE Unit, Itanagar, Arunachal Pradesh 791113, India

4 (presently) WWF-International, P.O. Box \# 7660, Baluwatar, Kathmandu, Nepal

Email: ${ }^{1}$ gopigv@gmail.com; ${ }^{3}$ bivash.pandav@ wwfnepal.org

plus web supplement of 2 pages

\begin{abstract}
This paper presents a comprehensive list of the birds of Bhitarkanika with special reference to globally threatened species occurring here. It is based, in large measure, on our observations throughout the protected area during August 2004 December 2006 together with unpublished records contributed by other observers. A total of 263 bird species belonging to 63 families have been recorded. A total of 79 birds are added to the existing checklist published in 1997. An account about one of India's largest breeding colony of water birds, and conservation importance of lesser known Sathbahaya wetland which serves as an important stopover for birds migrating to Chilka has also been mentioned.
\end{abstract}

KEYwORDS

Aquaculture, avifauna, Bhitarkanika mangroves, checklist, Chilka lake, heronry, Sathbahaya wetland

Assessment of the current status of bird species and habitat would benefit from the past bird surveys, natural history observations, community studies and abundance estimates. Ripley (1978) describes about the "relative scarcity" of avifauna when he visited the Simplipal hills after 28 years. It is believed that an annotated list of the Bhitarkanika birds complete so far as present knowledge permits will be of much value to future workers interested in this particular region. Much new matter in the form of hitherto unpublished field notes is included in this manuscript. A total of 263 birds belonging to 63 families were recorded of which 147 were resident, 99 were winter migrants, 15 were considered vagrants, 16 local migrants were summer migrants and the status of a few birds like Peregrine Falcon, Indian Skimmer, Booted Hawk Eagle, Black Eagle are yet to be ascertained.

Ornithological history in Indian mangroves and Bhitarkanika mangroves in particular

Very few studies have been so far carried out on the birds of Indian mangroves and Bhitarkanika in particular. Mukerjee (1969) studied the feeding habits of a few selected water birds in the mangrove forests of the Sunderbans. Prasad (1992) reports about a large inaccessible heronry in the Krishna mangroves in Andhra Pradesh where flamingoes, Spot-billed Pelicans, Spoonbills and Painted Storks nest together. Subramanya (1996) updated the existing information on the status, distribution and conservation of Indian heronries and listed Bhitarkanika as one of the oldest and largest heronry in the country. Chaudhari \& Chakrabarti (1973) studied the breeding biology of Openbill Stork, Little Cormorant and Large Egret. Several notes on species diversity and checklist of birds in different Indian mangroves like Thane Creek near Rutuchakkra Nature Park (Nitsure, 2002), Mahul Creek, Mumbai (Verma, 2002), Gulf of Kutch mangroves (Stanley, 2002), Pitchavaram mangroves (Sethuraman, 1997) were all published in different sources including symposia proceedings and newsletters. Samant (1985) recorded 121 species of birds in Ratnagiri mangroves in Maharashtra. Notes on the avifauna of Bhitarkanika mangroves occur at random, but an initial checklist of the birds of Bhitarkanika was attempted in the early 1990s by
Dani \& Kar (1992), and Pandav (1996). Pandav (1996) listed a total of 169 bird species from Bhitarkanika. Nayak (2003, 2005) studied the ecology of resident birds of the Bhitarkanika heronry and also the seasonality and occurrence of the kingfishers in the mangrove. Kalpana (2005) recently reported about the occurrence of Red-winged Crested Cuckoo in Bhitarkanika. Detailed ecological studies pertaining to breeding patterns, resource partitioning in terms of space and food and conservation assessment have been carried out recently in the Bhitarkanika heronry (Gopi, 2007; Gopi et al., 2007).

\section{Study AreA}

Geographically, Bhitarkanika is located between $20^{\circ} 30^{\prime}-$ $20^{\circ} 48^{\prime} \mathrm{N} \& 86^{\circ} 45^{\prime}-87^{\circ} 03^{\prime} \mathrm{E}$. It is the second largest existing mangrove ecosystem of India, which has much significance with regard to ecological, geomorphological and biological background that includes mangrove forests, rivers, creeks, estuaries, backwaters, accreted land and mud flats. Table 1 shows the areas of various wetland classes in Bhitarkanika. Bhitarkanika mangrove ecosystem flourishes in the deltaic region, formed by the rich alluvial deposits of Brahmani, Baitarani, Maipura and Dhamra rivers (Image $1^{\mathrm{w}}$ ).

Faunal significance includes the occurrence of a sizeable population of Estuarine Crocodile (Crocodylus porosus). Besides this, the Sanctuary is rich in other reptiles, birds and mammalian population. These mangrove forests are good habitat for King Cobra, Indian Python and Water Monitor Lizard. The annual rainfall ranges from 920-3000mm. Bhitarkanika represents one of the richest and most diversified mangrove floras in the country. About 62 species of mangrove and its associates are found in Bhitarkanika Sanctuary. Existence of three species of Rhizophora, Heritiera and Avicennia each and four species of Bruguiera are some of the interesting features of the flora of Bhitarkanika. The dominant genera of mangroves and its associates include Acanthus, Achrostichum, Aegialitis, Aglaia, Avicennia, Excoecaria, Brownlowia,

${ }^{\mathrm{w}}$ See Images in the web supplement at www.zoosprint.org

Manuscript 1716; (C) ZOO; Date of publication 21 September 2007 Received 10 February 2007; Revised received 23 July 2007; Finally accepted 01 September 2007; Edited by C. Srinivasulu 
Table 1. Various wetland classes and their area in Bhitarkanika

\begin{tabular}{llll}
\hline $\begin{array}{l}\text { Mangrove } \\
\text { in } \mathbf{~ k m}^{2}\end{array}$ & $\begin{array}{l}\text { Mud flat } \\
\text { in } \mathbf{~ k m}^{2}\end{array}$ & $\begin{array}{l}\text { Sandy area } \\
\text { in } \mathbf{~ k m}^{2}\end{array}$ & $\begin{array}{l}\text { Coastal Plantation } \\
\text { in } \mathbf{~ m}^{2}\end{array}$ \\
\hline 267.14 & 57.87 & 23.25 & 9.43 \\
\hline
\end{tabular}

Bruguiera, Ceriops, Rhizophora, Heritiera, Hibiscus, Kandelia, Lumnitzera, Phoenix, Sonneratia, Suaeda etc.

\section{MeTHODS}

The checklist is based primarily on field work conducted in Bhitarkanika mangroves (Image $1^{\mathrm{w}}$ ) from August 2004- December 2006. A total of 452 days were spent in the field observing bird diversity. Birds were observed from 0500- 1600hr with the help of a spotting scope and 8x40 Bushnell binoculars. During winters, all the major habitats were visited twice a week for monitoring wintering waterfowls. Surveys were conducted on foot in mangrove forests and agricultural fields, while in the rivers and small creeks birds were observed using mechanised motor boats and non-mechanised country boats, respectively.

In the heronry, total counts were carried out during the last week of August to enumerate the number of bird species nesting and number of nests of each species. Every tree was marked and bird species nesting and number of nests in each tree were recorded. Nests were identified with aid of parent bird or chicks. In absence of both, nest material and nest design were used to identify the species nesting. We have also included information from published literature, as cited in the text. Nomenclature follows Manakadan and Pittie (2002), except as noted.

The status (movement and seasonality of occurrence) and frequency of bird sightings in various habitats have been worked out basing on different parameters listed below.

Status: A taxon was accorded any one of the following: $\mathrm{R}$ Resident throughout the year; LM - Resident with some local movements; SM - Summer Migrant, MM - Monsoon Migrant; WM - Winter Migrant; V - Vagrant with only a single or couple of records, depending on its movement and seasonality of occurrence.

Frequency: A taxon was accorded any one of the following: A - Abundant, sighted on 9 to 10 out of 10 visits; C - Common, sighted on 6 to 8 out of 10 visits; O - Occasional, sighted on 3 to 5 out of 10 visits; R - Rare, sighted on less than 2 out of 10 visits, depending on the probability of sighting in the suitable habitat at the any given time.

Major Habitats: Different habitats where observations were carried out include; MF - Mangrove Forests; NMF NonMangrove Forest; OW - Open Wetlands; IZ - Intertidal Zones; TM - Tidal Mudflats; SB - Sand Bars; PF - Paddy Fields; AF Aquaculture Farms.

\section{RESUlts}

Globally threatened species in Bhitarkanika: Seventeen species of globally threatened species are found in Bhitarkanika mangroves and adjacent non mangrove wetlands (Table 2).

Spot-billed Pelican: This is a local migrant. About 15 birds were sighted in the heronry during July-August of 2005 and 2006. Highest number of 25 birds were recorded during 2002 (Nayak, 2003).
Table 2. List of globally threatened bird species in Bhitarkanika and their status according to IUCN/Birdlife International Red Data List 2006

\begin{tabular}{ll}
\hline Species & Category \\
\hline Spot-billed Pelican Pelecanus philippensis & VU \\
Lesser Adjutant Leptoptilos javanicus & VU \\
Painted Stork Mycteria leucocephala & NT \\
Black-necked Stork Ephippiorhynchus asiaticus & NT \\
Baer's Pochard Aythya baeri & VU \\
Pallas's Fish-eagle Haliaeetus leucoryphus & VU \\
White-rumped Vulture Gyps bengalensis & VR \\
Greater Spotted Eagle Aquila clanga & VU \\
Spotted Greenshank Tringa guttifer & EN \\
Brown-winged Kingfisher Halcyon amauroptera & NT \\
Indian Skimmer Rynchops albicollis & VU \\
Black-headed Ibis Threskiornis melanocephalus & NT \\
Darter Anhinga melanogaster & NT \\
Pallid Harrier Circus macrourus & NT \\
Pale-capped Pigeon Columba punicea & VU \\
Black-tailed Godwit Limosa limosa & NT \\
Black-bellied Tern Sterna acuticauda & NT \\
\hline
\end{tabular}

Black-necked Stork: A solitary bird is frequently spotted in the open meadows of Bhitarkanika forest block. During September 2005 a juvenile was spotted in the open meadows of Bhitarkanika forest block.

Baer's Pochard: Sighted twice; two birds were first sighted near Dobanthi (Barunei) in November 2005 and five birds were sighted in the Satabhaya intertidal wetlands on 8 November 2006.

White-backed Vulture: Approximate population of 10 individuals are present near Ragadapatia and Barapita forest block. Two old nests were located on Sonneratia apetala trees with three adults and two juveniles on 17 March 2005.

Spotted Greenshank: Two birds were sighted on 23 January 2003 in Udabali islands adjoining the sea and two birds were sighted on 10 December 2005 near Barunei river mouth.

Brown-winged Kingfisher: This species was sighted all over the National Park in the mangrove areas. An approximate population of 100 individuals are found here. It nests in the tree holes during July to September.

Black-headed Ibis: Breeds in the Bagagahan heronry. 136 nests were counted during the annual heronry census in August 2006. They built closely placed platform nests away from most of the species within the heronry forming subcolonies. Blackheaded Ibis had the mean largest clutch size of six per nest in the heronry.

Darter: (Image $2^{w}$ ) Breeds in the Bagagahan heronry. Over the years there is a marginal decline in the number of darters nesting in the heronry. Eighty-eight nests were counted during the annual heronry census in August 2006. They exhibited longer incubation duration (of more than 28 days) compared to other species in the heronry.

Indian Skimmer: (Image $3^{\mathrm{w}}$ ) Bhitarkanika is a major key congregation area for Indian skimmers in the country (Gopi et al., 2006) with more than 100 birds that frequent the Praharajpur and the Barunei estuary during the ebb tide for foraging. The seasonal movements of this species are not known. Though it is said to be a winter visitor to the Orissa coast, the fact that few individuals are sighted all through the year indicates that this species possibly breeds in Orissa.

Pallid Harrier: This is a winter migrant and rare to 
Bhitarkanika. This bird has been sighted thrice on $16^{-}$December 2005 and during last week of November 2006. On both occasions it was seen perched on Sonneratia apetala trees on the mud banks of the creeks.

Pale-capped Pigeon: Sighted twice during June - July 2003 feeding on Syzygium cuminii fruits.

Black-tailed Godwit: Local migrant, has been sighted almost throughout the year except during rainy season. Prefers paddy field, shallow water and sand bars during high tide. A total of 23,203 birds were counted during the Annual Mid-Winter Waterfowl Count conducted on 10 \& 11 January 2005.

Black-bellied Tern: Winter migrant. Birds have been observed near Dhamra estuary and on the sand bars of Coconut Wheeler Islands.

\section{Breeding notes}

One-hundred-and-four species of birds were observed to breed or showed evidence of breeding in Bhitarkanika. The mangrove forests of Bhitarkanika harbours one of the largest congregation of breeding water birds in the country and it is one of the top five largest heronries in India which hosts approximately 30,000 birds every year (Gopi \& Pandav, 2005; Subramanya, 1996; Chadha \& Kar, 1999). Eleven species of resident water birds, namely, Asian Openbill (Anastomus oscitans), Large Egret (Casmerodius albus), Intermediate Egret (Mesophoyx intermedia), Little Egret (Egretta garzetta), Cattle Egret (Bubulcus ibis), Grey Heron (Ardea cinerea), Purple Heron (Ardea purpurea) (Image 4w), Black-crowned Night Heron (Nycticorax nycticorax), Little Cormorant (Phalacrocorax niger), Darter (Anhinga melanogaster), and Black-headed Ibis (Threskiornis melanocephalus), breed in this heronry during the wet season (JuneDecember) (Table 3).

The heronry is located on an island covered with mangrove vegetation. The total area used for nesting is approximately tha. Five mangrove trees Excoecaria agallocha, Heritiera fomes, Cynometra iripa, Hibiscus tiliaecius and Tamarix troupii were used for nesting by the birds. Darters and Grey Herons were the first to arrive in the heronry during late June followed by Large Egrets, Purple Heron and Intermediate Egret (2005 \& 2006). Though Asian Openbill Storks arrived early, they delayed nesting till peak monsoons (2005). Night Heron and Black-headed Ibis were the last to arrive but they completed their nesting in a very short time of 3 to 4 days and started laying. The departure patterns of the birds followed the arrival sequence (2005 \& 2006). Birds start to leave the heronry by mid October and the entire herony is empty by second week of January (2005 \& 2006). Major nest materials that were used for nest building were derived from Excoecaria agallocha, Heritiera fomes, Hibiscus tiliaceus, Tamarix troupii, Cynometra iripa, Salvodora perscica, Salacia prinoides, Avivienia officianalis and Ceriops decandra and in one instance green leaves of Phoenix paludosa were also found in Darter's nest material.

A Lesser Adjutant Stork colony comprising of four nests on two Xylocarpus mekongensis trees were located in the Bhitarkanika forest block near Balijori creek. Nest building activity started in early October and the colony was active till mid January (Gopi \& Pandav, 2007).

Also a small colony comprising of eight Painted Stork nests were located near Ganjaikhia creek on three Heritira fomes trees. Nest height was about $7.5 \mathrm{~m}$. Nest building activity started in early September and the colony was active till the end of December (Gopi \& Pandav, 2007).

\section{Winter migrants}

A total of 98 species of winter visitors were recorded during the study in comparison to 57 species recorded earlier (Pandav, 1996). Two Spotted Greenshank (Tringa guttifer) were observed in the coastal mudflats near Barunei Chatka during low tide on 10 December 2005. Northern Pintail (Anas acuta) and Black-tailed Godwit (Limosa limosa) with approximate population of 41,928 and 23,203 recorded during the Annual Mid-Winter Waterfowl Count (2005). Approximately 882 Bar-headed Goose (Anser indicus) and 777 Northern Shoveller (Anas clypeata) were also recorded during the Annual Mid-Winter Waterfowl Count (2005).

\section{Sathabaya wetlands}

This lesser known wetland is the major bird habitat of Bhitarkanika. This wetland harbours 70,000-80,000 wintering species every year. Bar-headed Goose (Anser indicus), Brahminy Shelduck (Tadorna ferruginea), Eurasian Wigeon (Anas penelope.), Gadwall (A. strepera), Common Teal (A. crecca), Northern Pintail (A. acuta), Northern Shoveller (A. clypeata), Black-winged Stilt (Himantopus himantopus), Pacific Golden Plover (Pluvialis fulva), Whimbrel (Numenius phaeopus), Eurasian Curlew (N. arquata), Spotted Redshank (Tringa erythropus), Common Redshank ( $T$. totanus), Marsh Sandpiper (T. stagnatilis), Common Greenshank (T. nebularia), Little Stint (Calidris minuta), Temminck's Stint (C. temminckii), Curlew Sandpiper (C.ferruginea). Vast lands of paddy

Table 3. Number of nests enumerated year wise in the heronry from 1996-2006

\begin{tabular}{|c|c|c|c|c|c|c|c|c|c|c|c|}
\hline & 1996 & 1997 & 1998 & 1999 & 2000 & 2001 & 2002 & 2003 & 2004 & 2005 & 2006 \\
\hline Asian Openbill Stork & 8524 & 6843 & 6187 & 11349 & 6901 & 6906 & 6839 & 4986 & 6956 & 9061 & 7568 \\
\hline Large Egret & 527 & 1061 & 615 & 1624 & 231 & 231 & 1620 & 1305 & 2125 & 1205 & 1018 \\
\hline Intermediate Egret & 1703 & NA & NA & 236 & 146 & 146 & 1374 & 757 & 509 & 706 & 500 \\
\hline Little Egret & 478 & 36 & 120 & 276 & 12 & 12 & 313 & 211 & 234 & 61 & 59 \\
\hline Cattle Egret & 47 & NA & NA & 217 & 81 & 81 & 124 & 126 & 148 & 64 & 0 \\
\hline Grey Heron & 271 & 568 & 306 & 716 & 182 & 182 & 302 & 265 & 538 & 308 & 298 \\
\hline Purple Heron & 473 & 517 & 504 & 676 & 304 & 304 & 459 & 438 & 589 & 501 & 258 \\
\hline Night Heron & 390 & 399 & 505 & 572 & 96 & 95 & 510 & 353 & 540 & 372 & 363 \\
\hline Little Cormorant & 1960 & 734 & 1502 & 848 & 470 & 470 & 1777 & 1268 & 1937 & 1151 & 913 \\
\hline Darter & 225 & 92 & 106 & 200 & 126 & 126 & 234 & 219 & 316 & 123 & 128 \\
\hline Black-headed Ibis & 153 & 255 & 226 & 344 & 209 & 209 & 275 & 277 & 295 & 152 & 182 \\
\hline Total & 14751 & 10505 & 10071 & 17058 & 8758 & 8762 & 13827 & 10205 & 14187 & 13704 & 11287 \\
\hline
\end{tabular}

* NA - Not available 
Table 4. Annotated checklist of birds of Bhitarkanika mangroves, Orissa

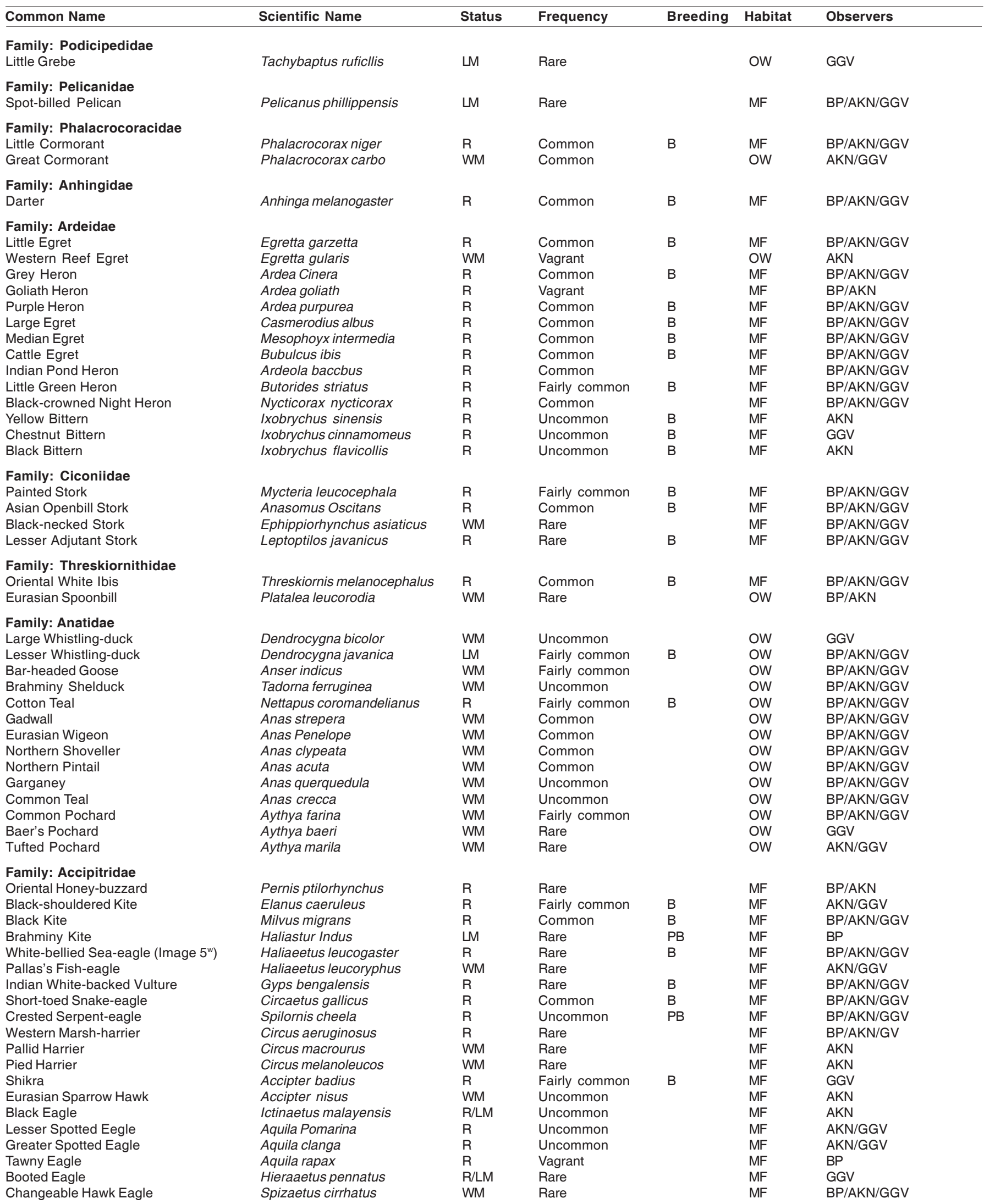




\begin{tabular}{|c|c|c|c|c|c|c|}
\hline Common Name & Scientific Name & Status & Frequency & Breeding & Habitat & Observers \\
\hline $\begin{array}{l}\text { Family: Pandionidae } \\
\text { Osprey }\end{array}$ & Pandion haliaetus & WM & Rare & & MF & \\
\hline $\begin{array}{l}\text { Family: Falconidae } \\
\text { Common Kestrel } \\
\text { Red-headed Falcon } \\
\text { Peregrine Falcon }\end{array}$ & $\begin{array}{l}\text { Falco tinnunculus } \\
\text { Falco chicquera } \\
\text { Falco peregrinus }\end{array}$ & $\begin{array}{l}\text { WM } \\
\text { LM } \\
\text { LM/WM }\end{array}$ & $\begin{array}{l}\text { Rare } \\
\text { Vagrant } \\
\text { Rare }\end{array}$ & & $\begin{array}{l}\text { MF } \\
\text { MF } \\
\text { MF }\end{array}$ & $\begin{array}{l}\text { AKN } \\
\text { GGV } \\
\text { AKN }\end{array}$ \\
\hline $\begin{array}{l}\text { Family: Phasinaidae } \\
\text { Grey Francolin } \\
\text { Red JungleFowl }\end{array}$ & $\begin{array}{l}\text { Francolinus pondicerianus } \\
\text { Gallus gallus }\end{array}$ & $\begin{array}{l}\mathrm{R} \\
\mathrm{R}\end{array}$ & $\begin{array}{l}\text { Vagrant } \\
\text { Common }\end{array}$ & $\mathrm{B}$ & $\begin{array}{l}\text { MF } \\
\text { MF }\end{array}$ & $\begin{array}{l}\text { AKN/GGV } \\
\text { BP/AKN/GGV }\end{array}$ \\
\hline $\begin{array}{l}\text { Family: Rallidae } \\
\text { Blue-breasted Rail } \\
\text { White-breasted Waterhen } \\
\text { Watercock } \\
\text { Purple Swamphen } \\
\text { Common Moorhen } \\
\text { Common Coot } \\
\text { Ruddy-breasted Crake }\end{array}$ & $\begin{array}{l}\text { Galliallus striatus } \\
\text { Amaurornis phoenucurus } \\
\text { Gallicrex cinerea } \\
\text { Porphyrio porphyorio } \\
\text { Gallinula chloropus } \\
\text { Fulica atra } \\
\text { Porzana fusca }\end{array}$ & $\begin{array}{l}R \\
R \\
R \\
R \\
R \\
\text { WM } \\
R\end{array}$ & $\begin{array}{l}\text { Uncommon } \\
\text { Fairly common } \\
\text { Common } \\
\text { Uncommon } \\
\text { Uncommon } \\
\text { Rare } \\
\text { Uncommon }\end{array}$ & $\begin{array}{l}\text { PB } \\
B\end{array}$ & $\begin{array}{l}\text { MF } \\
\text { MF } \\
\text { AF } \\
\text { MF } \\
\text { MF } \\
\text { OW } \\
\text { MF }\end{array}$ & $\begin{array}{l}\text { BP } \\
\text { BP/AKN/GGV } \\
\text { AKN/GGV } \\
\text { BP } \\
\text { BP/AKN } \\
\text { AKN } \\
\text { AKN }\end{array}$ \\
\hline $\begin{array}{l}\text { Family: Jacanidae } \\
\text { Pheasant-tailed Jacana } \\
\text { Bronze-winged Jacana }\end{array}$ & $\begin{array}{l}\text { Hydrophasianus chirurgus } \\
\text { Metopidius indicus }\end{array}$ & $\begin{array}{l}\mathrm{R} \\
\mathrm{R}\end{array}$ & $\begin{array}{l}\text { Uncommon } \\
\text { Fairly common }\end{array}$ & $\mathrm{B}$ & $\begin{array}{l}\text { MF } \\
\text { MF }\end{array}$ & $\begin{array}{l}\text { AKN/GGV } \\
\text { BP/AKN/GGV }\end{array}$ \\
\hline $\begin{array}{l}\text { Family: Rostratulidae } \\
\text { Greater Painted Snipe }\end{array}$ & Rostratula benghalensis & $\mathrm{R}$ & Uncommon & & MF & GGV \\
\hline $\begin{array}{l}\text { Family: Haematopodidae } \\
\text { Eurasian Oystercatcher }\end{array}$ & Haematopus ostralegus & WM & Common & & SB & AKN/GG V \\
\hline $\begin{array}{l}\text { Family: Charadriidae } \\
\text { Pacific Golden-plover } \\
\text { Grey Plover } \\
\text { Common Ringed Plover } \\
\text { Little Ringed Lover } \\
\text { Kentish Plover } \\
\text { Lesser Sand Plover } \\
\text { Greater Sand Plover } \\
\text { Yellow-wattled Lapwing } \\
\text { Grey-headed Lapwing } \\
\text { Red-wattled Lapwing } \\
\text { White tailed Lapwing }\end{array}$ & $\begin{array}{l}\text { Pluvialis fulva } \\
\text { Pluvialis squatarola } \\
\text { Charadrius hiaticula } \\
\text { Charadrius dubius } \\
\text { Charadrius alexandrinus } \\
\text { Charadrius mongolus } \\
\text { Charadrius leschenaultii } \\
\text { Vanellus malarbaricus } \\
\text { Vanellus cinereus } \\
\text { Vanellus indicus } \\
\text { Vanellus leucurus }\end{array}$ & $\begin{array}{l}\text { WM } \\
\text { WM } \\
\text { WM } \\
\text { WM } \\
\text { WM } \\
\text { WM } \\
\text { WM } \\
\text { R } \\
\text { WM } \\
\text { R } \\
\text { WM }\end{array}$ & $\begin{array}{l}\text { Fairly common } \\
\text { Rare } \\
\text { Uncommon } \\
\text { Fairly common } \\
\text { Uncommon } \\
\text { Common } \\
\text { Rare } \\
\text { Uncommon } \\
\text { Fairly common } \\
\text { Common } \\
\text { Rare }\end{array}$ & B & $\begin{array}{l}\text { TMF } \\
\text { TMF } \\
\text { TMF } \\
\text { TMF } \\
\text { TMF } \\
\text { TMF } \\
\text { TMF } \\
\text { MF } \\
\text { MF } \\
\text { MF } \\
\text { MF }\end{array}$ & $\begin{array}{l}\text { AKN/GGV } \\
\text { AKN } \\
\text { AKN } \\
\text { BP/AKN/GGV } \\
\text { BP/AKN/GGV } \\
\text { BP/AKN/GGV } \\
\text { BP/AKN/GGV } \\
\text { BP/AKN/GGV } \\
\text { BP/AKN/GGV } \\
\text { BP/AKN/GGV } \\
\text { AKN }\end{array}$ \\
\hline $\begin{array}{l}\text { Family: Scolopacidae } \\
\text { Pintail Snipe } \\
\text { Common Snipe } \\
\text { Jack Snipe } \\
\text { Black-tailed Godwit } \\
\text { Bar-tailed Godwit } \\
\text { Whimbrel } \\
\text { Eurasian Curlew } \\
\text { Spotted Redshank } \\
\text { Common Redshank } \\
\text { Marsh Sandpiper } \\
\text { Common Greenshank } \\
\text { Spotted Greenshank } \\
\text { Green Sandpiper } \\
\text { Wood Sandpiper } \\
\text { Terek Sandpiper } \\
\text { Common Sandpiper } \\
\text { Ruddy Turnstone } \\
\text { Red Knot } \\
\text { Sanderling } \\
\text { Little Stint } \\
\text { Temminck's Stint } \\
\text { Long toed Stint } \\
\text { Dunlin } \\
\text { Curlew Sandpiper } \\
\text { Ruff }\end{array}$ & $\begin{array}{l}\text { Gallinago stenura } \\
\text { Gallinago gallinago } \\
\text { Lymnocryptes minimus } \\
\text { Limosa limosa } \\
\text { Limosa lapponica } \\
\text { Numenius phaeopus } \\
\text { Numenius arquata } \\
\text { Tringa erythropus } \\
\text { Tringa tetanus } \\
\text { Tringa stagnatilis } \\
\text { Tringa nebularia } \\
\text { Tringa guttifer } \\
\text { Tringa ochropus } \\
\text { Tringa glareola } \\
\text { Tringa terek } \\
\text { Tringa hypoleucos } \\
\text { Arenaria interpres } \\
\text { Calidris cautus } \\
\text { Calidris alba } \\
\text { Calidris minuta } \\
\text { Calidris temminckii } \\
\text { Calidris subminuta } \\
\text { Calidris alpine } \\
\text { Calidris testacea } \\
\text { Philomachus pugnax }\end{array}$ & $\begin{array}{l}\text { WM } \\
\text { WM } \\
\text { WM } \\
\text { R } \\
\text { WM } \\
\text { R } \\
\text { WM } \\
\text { WM } \\
\text { WM } \\
\text { WM } \\
\text { WM } \\
\text { WM } \\
\text { WM } \\
\text { WM } \\
\text { WM } \\
\text { WM } \\
\text { WM } \\
\text { WM } \\
\text { WM } \\
\text { WM } \\
\text { WM } \\
\text { WM } \\
\text { WM } \\
\text { WM } \\
\text { WM }\end{array}$ & $\begin{array}{l}\text { Fairly Common } \\
\text { Rare } \\
\text { Uncommon } \\
\text { Common } \\
\text { Rare } \\
\text { Common } \\
\text { Common } \\
\text { Rare } \\
\text { Fairly Common } \\
\text { Uncommon } \\
\text { Common } \\
\text { Rare } \\
\text { Rare } \\
\text { Fairly Common } \\
\text { Fairly Common } \\
\text { Common } \\
\text { Rare } \\
\text { Rare } \\
\text { Rare } \\
\text { Common } \\
\text { Uncommon } \\
\text { Rare } \\
\text { Uncommon } \\
\text { Fairly Common } \\
\text { Common }\end{array}$ & & $\begin{array}{l}\text { OW } \\
\text { OW } \\
\text { OW } \\
\text { OW } \\
\text { OW } \\
\text { OW } \\
\text { OW } \\
\text { OW } \\
\text { OW } \\
\text { OW } \\
\text { OW } \\
\text { OW } \\
\text { OW } \\
\text { OW } \\
\text { OW } \\
\text { OW } \\
\text { TM } \\
\text { OW } \\
\text { OW } \\
\text { OW } \\
\text { OW } \\
\text { OW } \\
\text { OW } \\
\text { OW }\end{array}$ & $\begin{array}{l}\text { BP } \\
\text { AKN } \\
\text { AKN } \\
\text { BP/AKN/GGV } \\
\text { BP/AKN/GGV } \\
\text { BP/AKN/GGV } \\
\text { BP/AKN/GGV } \\
\text { AKN } \\
\text { BP/AKN/GGV } \\
\text { AKN } \\
\text { BP/AKN/GGV } \\
\text { GGV } \\
\text { BP/AKN/GGV } \\
\text { BP/AKN/GGV } \\
\text { BP/AKN/GGV } \\
\text { BP/AKN/GGV } \\
\text { BP/AKN } \\
\text { AKN } \\
\text { AKN/GGV } \\
\text { BP } \\
\text { BP } \\
\text { AKN } \\
\text { BP/GGV } \\
\text { AKN/GGV } \\
\text { BP/GGV }\end{array}$ \\
\hline $\begin{array}{l}\text { Family: Recurvirostridae } \\
\text { Blac-winged Stilt } \\
\text { Pied Avocet }\end{array}$ & $\begin{array}{l}\text { Himantopus himantopus } \\
\text { Recurvirostra avosetta }\end{array}$ & $\begin{array}{l}\text { LM } \\
\text { WM }\end{array}$ & $\begin{array}{l}\text { Common } \\
\text { Fairly common }\end{array}$ & PB & $\begin{array}{l}\text { OW } \\
\text { SB }\end{array}$ & $\begin{array}{l}\text { BP/AKN/GGV } \\
\text { BP/AKN/GGV }\end{array}$ \\
\hline $\begin{array}{l}\text { Family: Dromadidae } \\
\text { Crab-plover }\end{array}$ & Dromas ardeola & WM & Rare & & TMF & AKN/GGV \\
\hline $\begin{array}{l}\text { Family: Burhinidae } \\
\text { Stone-curlew } \\
\text { Great Stone-plover }\end{array}$ & $\begin{array}{l}\text { Burhinus oedicnemus } \\
\text { Esacus recurvirostris }\end{array}$ & $\begin{array}{l}\text { WM } \\
\text { WM }\end{array}$ & $\begin{array}{l}\text { Rare } \\
\text { Rare }\end{array}$ & & $\begin{array}{l}\text { TMF } \\
\text { TMF }\end{array}$ & $\begin{array}{l}\text { GGV } \\
\text { BP/AKN/GGV }\end{array}$ \\
\hline
\end{tabular}




\begin{tabular}{|c|c|c|c|c|c|c|}
\hline Common Name & Scientific Name & Status & Frequency & Breeding & Habitat & Observers \\
\hline \multicolumn{7}{|l|}{ Family: Glareolidae } \\
\hline Oriental Pratincole & Glareola maldivarum & WM & Rare & & MF & $\mathrm{BP} / \mathrm{AKN} / \mathrm{GGV}$ \\
\hline Small Pratincole & Glareola lacteal & WM & Rare & & MF & AKN/GGV \\
\hline \multicolumn{7}{|l|}{ Family: Laridae } \\
\hline Heuglin's Gull & Larus beuglini & WM & Common & & SB & $\mathrm{BP} / \mathrm{AKN} / \mathrm{GGV}$ \\
\hline Pallas's Gull & Larus ichthyaetus & WM & Common & & SB & $\mathrm{BP} / \mathrm{AKN} / \mathrm{GGV}$ \\
\hline Brow- headed Gull & (Larus brunnicephalus & WM & Common & & SB & $\mathrm{BP} / \mathrm{AKN} / \mathrm{GGV}$ \\
\hline Black-headed Gull & Larus ridibundus & WM & Fairly common & & SB & $\mathrm{BP} / \mathrm{AKN} / \mathrm{GGV}$ \\
\hline Gull-billed Tern & Gelochelidon nilotica & WM & Uncommon & & SB & $\mathrm{BP} / \mathrm{AKN} / \mathrm{GGV}$ \\
\hline Capsian Tern & Hydroprogne caspia & WM & Rare & & SB & GGV \\
\hline River Tern & Sterna aurantia & பM & Rare & & SB & $\mathrm{BP} / \mathrm{AKN} / \mathrm{GGV}$ \\
\hline Lesser Crested Tern & Sterna bengalensis & WM & Uncommon & & SB & $\mathrm{BP} / \mathrm{AKN} / \mathrm{GGV}$ \\
\hline Large Crested Tern & Sterna bergii & WM & Rare & & SB & GGV \\
\hline Black-bellied Terns & Sterna acuticauda & WM & Rare & PB & SB & $\mathrm{BP} / \mathrm{AKN} / \mathrm{GGV}$ \\
\hline Whiskered Tern & Chlidonias hybrida & LM & Fairly common & & SB & GGV \\
\hline \multicolumn{7}{|l|}{ Family: Rynchopidae } \\
\hline Indian Skimmer & Rynchops albicollis & $\mathrm{R} / \mathrm{WM}$ & Common & & & GGV/AKN \\
\hline \multicolumn{7}{|l|}{ Family: Columbidae } \\
\hline Blue Rock Pigeon & Columba livia & $\mathrm{R}$ & Common & $B$ & MF & $\mathrm{BP} / \mathrm{AKN} / \mathrm{GGV}$ \\
\hline Purple Wood Pigeon & Columba punicea & $\mathrm{R}$ & Rare & & MF & \\
\hline Oriental Turtle-dove & Streptopelia orientalis & $\mathrm{R}$ & Common & & MF & $\mathrm{BP} / \mathrm{AKN} / \mathrm{GGV}$ \\
\hline Spotted Dove & Streptopelia chinensis & $\mathrm{R}$ & Common & $\mathrm{B}$ & MF & $\mathrm{BP} / \mathrm{AKN} / \mathrm{GGV}$ \\
\hline Eurasian Collared-dove & Streptopelia decaocto & $\mathrm{R}$ & Common & $\mathrm{B}$ & MF & $\mathrm{BP} / \mathrm{AKN} / \mathrm{GGV}$ \\
\hline Emerald Dove & Chalcophaps indica & $\mathrm{R}$ & Common & B & MF & $\mathrm{BP} / \mathrm{AKN} / \mathrm{GGV}$ \\
\hline Orange-breasted Green Pigeon & Treron bicincta & $\mathrm{R}$ & Common & $\mathrm{B}$ & MF & $\mathrm{BP} / \mathrm{AKN} / \mathrm{GGV}$ \\
\hline \multicolumn{7}{|l|}{ Family: Psittacidae } \\
\hline Alexandrine Parakeet & Psittacula eupatria & $\mathrm{R}$ & Rare & & MF & AKN/GGV \\
\hline Rose-ringed Parakeet & Psittacula krameri & $\mathrm{R}$ & Common & B & MF & $\mathrm{BP} / \mathrm{AKN} / \mathrm{GGV}$ \\
\hline Blossom-headed Parakeet & Psittacula roseate & $\mathrm{R}$ & Vagrant & & MF & GGV \\
\hline \multicolumn{7}{|l|}{ Family: Cuculidae } \\
\hline Pied Crested Cuckoo & Clamator jacobinus & MM & Rare & & MF & $\mathrm{BP} / \mathrm{AKN} / \mathrm{GGV}$ \\
\hline Red-winged Cuckoo & Clamator coromandus & PM & Uncommon & & MF & $\mathrm{BP} / \mathrm{KA}$ \\
\hline Brainfever Bird & Hierococcyx varius & $\mathrm{R}$ & Common & $\mathrm{B}$ & MF & $\mathrm{BP} / \mathrm{AKN} / \mathrm{GGV}$ \\
\hline Common Cuckoo & Cuculus canorus & LM & Uncommon & & MF & AKN/GGV \\
\hline Indian Cuckoo & Cuculus micropterus & $\mathrm{R}$ & Vagrant & & MF & GGV \\
\hline Indian Plaintive Cuckoo & Cacomantis passerinus & $\mathrm{R}$ & Vagrant & & MF & GGV \\
\hline Asian Koel & Eudynamys scolopacea & $\mathrm{R}$ & Common & $\mathrm{B}$ & MF & $\mathrm{BP} / \mathrm{AKN} / \mathrm{GGV}$ \\
\hline Large Green-billed Malkoha & Phaenicophaeus viridirostris & $\mathrm{R}$ & Fairly common & B & MF & $\mathrm{BP} / \mathrm{AKN} / \mathrm{GGV}$ \\
\hline Greater Coucal & Centropus sinensis & $\mathrm{R}$ & Common & B & $\mathrm{MF}$ & $\mathrm{BP} / \mathrm{AKN} / \mathrm{GGV}$ \\
\hline \multicolumn{7}{|l|}{ Family: Tytonidae } \\
\hline Barn Owl & Tyto alba & $\mathrm{R}$ & Rare & & MF & AKN \\
\hline \multicolumn{7}{|l|}{ Family: Strigidae } \\
\hline Oriental Scops-owl & Otus scops & $\mathrm{R}$ & Vagrant & & MF & GGV \\
\hline Collared Scops Owl & Otus bakkamoena & $\mathrm{R}$ & Common & PB & MF & $\mathrm{BP} / \mathrm{AKN} / \mathrm{GGV}$ \\
\hline Brown Fish-owl & Ketupa zeylonensis & $\mathrm{R}$ & Rare & & MF & AKN \\
\hline Spotted Owlet & Athene brama & $\mathrm{R}$ & Common & B & MF & $\mathrm{BP}$ \\
\hline \multicolumn{7}{|l|}{ Family: Caprimulgidae } \\
\hline Indian Jungle Nightjar & Caprimulgus indicus & $\mathrm{R}$ & Common & $\mathrm{B}$ & MF & $\mathrm{BP} / \mathrm{AKN} / \mathrm{GGV}$ \\
\hline Large-tailed Nightjar & Caprimulgus macrurus & $\mathrm{R}$ & Common & B & MF & $\mathrm{BP} / \mathrm{AKN} / \mathrm{GGV}$ \\
\hline \multicolumn{7}{|l|}{ Family: Apodidae } \\
\hline Asian Palm-swift & Cypsiurus parvus & $\mathrm{R}$ & Common & B & MF & GGV \\
\hline House Swift & Apus affinis & $\mathrm{R}$ & Common & B & MF & GGV \\
\hline \multicolumn{7}{|l|}{ Family: Alcedinidae } \\
\hline Small Blue Kingfisher & Alcedo atthis & $\mathrm{R}$ & Common & $\mathrm{B}$ & MF & $\mathrm{BP} / \mathrm{AKN} / \mathrm{GGV}$ \\
\hline Stork-billed Kingfisher & Halcyon capensis & $\mathrm{R}$ & Rare & $\mathrm{B}$ & MF & AKN/GGV \\
\hline Lesser pied Kingfisher & Ceryle rudis & $\mathrm{R}$ & Common & B & OMF & $\mathrm{BP} / \mathrm{AKN} / \mathrm{GGV}$ \\
\hline Brown-winged Kingfisher & Halcyon amauroptera & WM & Common & B & MF & $\mathrm{BP} / \mathrm{AKN} / \mathrm{GGV}$ \\
\hline Ruddy Kingfisher & Halcyon coromanda & $\mathrm{R}$ & Vagrant & & MF & AKN \\
\hline White-breasted Kingfisher & Halcyon smyrnensis & $\mathrm{R}$ & Common & $\mathrm{B}$ & MF & $\mathrm{BP} / \mathrm{AKN} / \mathrm{GGV}$ \\
\hline Black-capped Kingfisher & Helcyon pileata & LM & Common & & MF & $\mathrm{BP} / \mathrm{AKN} / \mathrm{GGV}$ \\
\hline Collared Kingfisher (Image $6^{w}$ ) & Todiramphus chloris & $\mathrm{R}$ & Common & B & MF & $\mathrm{BP} / \mathrm{AKN} / \mathrm{GGV}$ \\
\hline Family: Meropidae & & & & & & \\
\hline Small Bee-eater & Merops orientalis & $\mathrm{R}$ & Common & $\mathrm{B}$ & MF & $\mathrm{BP} / \mathrm{AKN} / \mathrm{GGV}$ \\
\hline Blue Tailed Bee Eater & Merops phillipinus & SM & Fairly Common & $\mathrm{B}$ & MF & $\mathrm{BP} / \mathrm{AKN} / \mathrm{GGV}$ \\
\hline Chestnut-headed Bee Eater & Merops leschenaulti & LM & Vagrant & & MF & \\
\hline Family: Coraciidae & & & & & & \\
\hline Indian Roller & Coracias benghalensis & $\mathrm{R}$ & Fairly common & B & MF & $\mathrm{BP} / \mathrm{AKN} / \mathrm{GGV}$ \\
\hline Family: Upupidae & & & & & & \\
\hline Common Hoopoe & Upupa epops & $\underline{R}$ & Fairly Common & $\mathrm{B}$ & $\mathrm{MF}$ & $\mathrm{BP} / \mathrm{AKN} / \mathrm{GGV}$ \\
\hline
\end{tabular}




\begin{tabular}{|c|c|c|c|c|c|c|}
\hline Common Name & Scientific Name & Status & Frequency & Breeding & Habitat & Observers \\
\hline $\begin{array}{l}\text { Family: Bucerotidae } \\
\text { Indian Grey Hornbill }\end{array}$ & Ocyceros birostris & $\mathrm{R}$ & Common & $\mathrm{B}$ & MF & $\mathrm{BP} / \mathrm{AKN} / \mathrm{GGV}$ \\
\hline $\begin{array}{l}\text { Family: Capitonidae } \\
\text { Coppersmith Barbet }\end{array}$ & Megalaima haemacephala & $r$ & Common & $\mathrm{B}$ & MF & $\mathrm{BP} / \mathrm{AKN} / \mathrm{GGV}$ \\
\hline $\begin{array}{l}\text { Family: Picidae } \\
\text { Eurasian Wryneck } \\
\text { Speckled Piculet } \\
\text { Rufous Woodpecker } \\
\text { Brown-capped Pygmy Wood Pecker } \\
\text { Fulvous Breasted Pied Woodpecker } \\
\text { Black-naped Green Woodpecker } \\
\text { Lesser Golden-backed Woodpecker } \\
\text { Greater Golden-backed Woodpecker }\end{array}$ & $\begin{array}{l}\text { Jynx torquilla } \\
\text { Picumnus innominatus } \\
\text { Celeus brachyurus } \\
\text { Dendrocopos nanus } \\
\text { Dendrocopos macei } \\
\text { Picus canus } \\
\text { Dinopium benghalense } \\
\text { Chrysocolaptes lucidus }\end{array}$ & $\begin{array}{l}\text { WM } \\
R \\
R \\
\text { LM } \\
R \\
R \\
R \\
R\end{array}$ & $\begin{array}{l}\text { Rare } \\
\text { Rare } \\
\text { Fairly common } \\
\text { Rare } \\
\text { Uncommon } \\
\text { Fairly common } \\
\text { Common } \\
\text { Common }\end{array}$ & $\begin{array}{l}\text { B } \\
\text { B } \\
\text { B }\end{array}$ & $\begin{array}{l}\text { MF } \\
\text { MF } \\
\text { MF } \\
\text { MF } \\
\text { MF } \\
\text { MF } \\
\text { MF } \\
\text { MF }\end{array}$ & $\begin{array}{l}\text { AKN } \\
\text { AKN } \\
\text { BP } \\
\text { GGV } \\
\text { BP } \\
\text { BP/AKN/GGV } \\
\text { BP/AKN/GGV } \\
\text { BP/AKN/GGV }\end{array}$ \\
\hline $\begin{array}{l}\text { Family: Pittidae } \\
\text { Indian Pitta } \\
\text { Mangrove Pitta }\end{array}$ & $\begin{array}{l}\text { Pitta brachyura } \\
\text { Pitta megarhyncha }\end{array}$ & $\begin{array}{l}\mathrm{LM} \\
\mathrm{R}\end{array}$ & $\begin{array}{l}\text { Fairly common } \\
\text { Fairly common }\end{array}$ & $\mathrm{B}$ & & $\begin{array}{l}\mathrm{BP} \\
\mathrm{AKN} / \mathrm{GGV}\end{array}$ \\
\hline $\begin{array}{l}\text { Family: Alaudidae } \\
\text { Red-winged Bush-lark } \\
\text { Bengal Bush Lark } \\
\text { Ashy Crowned Sparrow Lark } \\
\text { Greater Short-toed Lark } \\
\text { Common Crested Lark } \\
\text { Eastern Sky Lark }\end{array}$ & $\begin{array}{l}\text { Mirafra erythroptera } \\
\text { Mirafra assamica } \\
\text { Eremopterix grisea } \\
\text { Calandrella brachydactyla } \\
\text { Galerida cristata } \\
\text { Alauda gulgula }\end{array}$ & $\begin{array}{l}\text { R } \\
\text { WM } \\
R \\
R \\
R \\
R\end{array}$ & $\begin{array}{l}\text { Common } \\
\text { Rare } \\
\text { Fairly common } \\
\text { Vagrant } \\
\text { Fairly common } \\
\text { Fairly common }\end{array}$ & $\mathrm{B}$ & $\begin{array}{l}\text { MF } \\
\text { MF } \\
\text { MF } \\
\text { MF } \\
\text { MF } \\
\text { MF }\end{array}$ & $\begin{array}{l}\text { AKN } \\
\text { AKN } \\
\text { BP/AKN/GGV } \\
\text { GGV } \\
\text { AKN } \\
\text { AKN }\end{array}$ \\
\hline $\begin{array}{l}\text { Family: Hirundinidae } \\
\text { Common Swallow } \\
\text { Red-rumped Swallow } \\
\text { Asian House-martin }\end{array}$ & $\begin{array}{l}\text { Hirundo rustica } \\
\text { Hirundo daurica } \\
\text { Delichon dasypus }\end{array}$ & $\begin{array}{l}\text { WM } \\
\text { WM } \\
\text { WM }\end{array}$ & $\begin{array}{l}\text { Common } \\
\text { Fairly common } \\
\text { Uncommon }\end{array}$ & & $\begin{array}{l}\text { MF } \\
\text { MF } \\
\text { MF }\end{array}$ & $\begin{array}{l}\text { BP/AKN/GGV } \\
\text { BP/AKN/GGV } \\
\text { GGV }\end{array}$ \\
\hline $\begin{array}{l}\text { Family: Motacilidae } \\
\text { Forest Wagtail } \\
\text { White Wagtail } \\
\text { Citrine Wagtail } \\
\text { Yellow Wagtail } \\
\text { Grey Wagtail } \\
\text { Richard's Pipit } \\
\text { Paddyfield Pipit } \\
\text { Tawny Pipit } \\
\text { Eurasian Tree Pipit } \\
\text { Oriental Tree Pipit }\end{array}$ & $\begin{array}{l}\text { Dendronanthus indicus } \\
\text { Motacilla alba } \\
\text { Motacilla cireola } \\
\text { Motacilla flava } \\
\text { Motacilla cinerea } \\
\text { Anthus richardi } \\
\text { Anthus rufulus } \\
\text { Anthus campestris } \\
\text { Anthus trivialis } \\
\text { Anthus hodgsoni }\end{array}$ & $\begin{array}{l}\text { WM } \\
\text { WM } \\
\text { WM } \\
\text { WM } \\
\text { WM } \\
\text { R } \\
\text { R } \\
\text { WM } \\
\text { WM } \\
\text { WM }\end{array}$ & $\begin{array}{l}\text { Fairly common } \\
\text { Uncommon } \\
\text { Fairly common } \\
\text { Fairly common } \\
\text { Uncommon } \\
\text { Uncommon } \\
\text { Common } \\
\text { Uncommon } \\
\text { Rare } \\
\text { Uncommon }\end{array}$ & B & $\begin{array}{l}\text { MF } \\
\text { MF } \\
\text { MF } \\
\text { MF } \\
\text { MF } \\
\text { MF/PF } \\
\text { MF/PF } \\
\text { MF } \\
\text { MF } \\
\text { MF }\end{array}$ & $\begin{array}{l}\text { BP/AKN/GGV } \\
\text { AKN } \\
\text { BP/AKN } \\
\text { AKN } \\
\text { AKN } \\
\text { GGV } \\
\text { BP/AKN/GGV } \\
\text { AKN } \\
\text { AKN } \\
\text { BP/AKN/GGV }\end{array}$ \\
\hline $\begin{array}{l}\text { Family: Campephagidae } \\
\text { Large Cuckooshrike } \\
\text { Black Winged Cuckoo-shrike } \\
\text { Black-headed Cuckoo-shrike } \\
\text { Ashy Minivet } \\
\text { Small Minivet }\end{array}$ & $\begin{array}{l}\text { Coracina macei } \\
\text { Coracina melaschistos } \\
\text { Coracina melanoptera } \\
\text { Perricrocotus divaricatus } \\
\text { Pericrocotus cinnamomeus }\end{array}$ & $\begin{array}{l}\text { R } \\
R \\
R \\
\text { WM } \\
\text { R }\end{array}$ & $\begin{array}{l}\text { Vagrant } \\
\text { Rare } \\
\text { Fairly common } \\
\text { Rare } \\
\text { Fairly common }\end{array}$ & B & $\begin{array}{l}\text { MF } \\
\text { MF } \\
\text { MF } \\
\text { MF } \\
\text { MF }\end{array}$ & $\begin{array}{l}\text { GGV } \\
\text { BP/AKN/GGV } \\
\text { BP/AKN/GGV } \\
\text { AKN } \\
\text { BP/AKN/GGV }\end{array}$ \\
\hline $\begin{array}{l}\text { Family: Pycnonotidae } \\
\text { Red-whiskered Bulbul } \\
\text { Red-vented Bulbul }\end{array}$ & $\begin{array}{l}\text { Pycnonotus jocosus } \\
\text { Pycnonotus cafer }\end{array}$ & $\begin{array}{l}\mathrm{R} \\
\mathrm{R}\end{array}$ & $\begin{array}{l}\text { Fairly common } \\
\text { Fairly common }\end{array}$ & $\begin{array}{l}\mathrm{B} \\
\mathrm{B}\end{array}$ & $\begin{array}{l}\text { MF } \\
\text { MF }\end{array}$ & $\begin{array}{l}\mathrm{BP} / \mathrm{AKN} / \mathrm{GGV} \\
\mathrm{AKN} / \mathrm{GGV}\end{array}$ \\
\hline $\begin{array}{l}\text { Family: Irenidae } \\
\text { Common Iora } \\
\text { Jerdon's Chloropsis }\end{array}$ & $\begin{array}{l}\text { Aegithina tiphia } \\
\text { Chloropsis cochinchinensis }\end{array}$ & $\begin{array}{l}\mathrm{R} \\
\mathrm{WM}\end{array}$ & $\begin{array}{l}\text { Common } \\
\text { Vagrant }\end{array}$ & B & $\begin{array}{l}\text { MF } \\
\text { MF }\end{array}$ & $\begin{array}{l}\text { AKN/GGV } \\
\text { AKN }\end{array}$ \\
\hline $\begin{array}{l}\text { Family: Laniidae } \\
\text { Rufos-backed Shrike } \\
\text { Brown Shrike }\end{array}$ & $\begin{array}{l}\text { Lanius schach } \\
\text { Lanius cristatus }\end{array}$ & $\begin{array}{l}\text { WM } \\
\mathrm{R}\end{array}$ & $\begin{array}{l}\text { Vagrant } \\
\text { Uncommon }\end{array}$ & & $\begin{array}{l}\text { MF } \\
\text { MF }\end{array}$ & $\begin{array}{l}\text { GGV } \\
\mathrm{BP}\end{array}$ \\
\hline $\begin{array}{l}\text { Family: Turdinae } \\
\text { Orange Headed Ground Thrush } \\
\text { Tickel's Thrush } \\
\text { Oriental Magpie-robin } \\
\text { Black Redstart }\end{array}$ & $\begin{array}{l}\text { Zoothera citrina } \\
\text { Turdus unicolor } \\
\text { Copsychus saularis } \\
\text { Phoenicurus ochruros }\end{array}$ & $\begin{array}{l}\mathrm{R} \\
\mathrm{WM} \\
\mathrm{R} \\
\mathrm{R}\end{array}$ & $\begin{array}{l}\text { Fairly common } \\
\text { Rare } \\
\text { Common } \\
\text { Rare }\end{array}$ & B & $\begin{array}{l}\text { MF } \\
\text { MF } \\
\text { MF } \\
\text { MF }\end{array}$ & $\begin{array}{l}\text { BP/AKN/GGV } \\
\text { BP/AKN/GGV } \\
\text { BP/AKN/GGV } \\
\text { BP/AKN/GGV }\end{array}$ \\
\hline $\begin{array}{l}\text { Family: Timalinae } \\
\text { Common Babbler } \\
\text { Jungle Babbler } \\
\text { Striated Babbler } \\
\text { Yellow Breasted Babbler } \\
\text { Red-capped Babbler }\end{array}$ & $\begin{array}{l}\text { Turdoides caudatus } \\
\text { Turdoides striatus } \\
\text { Turdoides earlei } \\
\text { Macronous gularis } \\
\text { Timalia pileata }\end{array}$ & $\begin{array}{l}\mathrm{R} \\
\mathrm{R} \\
\mathrm{R} \\
\mathrm{R} \\
\mathrm{R}\end{array}$ & $\begin{array}{l}\text { Common } \\
\text { Common } \\
\text { Fairly common } \\
\text { Fairly common } \\
\text { Uncommon }\end{array}$ & $\begin{array}{l}\mathrm{B} \\
\mathrm{B}\end{array}$ & $\begin{array}{l}\text { MF } \\
\text { MF } \\
\text { MF } \\
\text { MF } \\
\text { MF }\end{array}$ & $\begin{array}{l}\text { BP/AKN/GGV } \\
\text { BP/AKN/GGV } \\
\text { AKN } \\
\text { BP/AKN } \\
\text { BP/AKN }\end{array}$ \\
\hline $\begin{array}{l}\text { Family: Sylviinae } \\
\text { Streaked Fantail-warbler } \\
\text { Yellow-bellied Prinia } \\
\text { Plain Prinia } \\
\text { Ashy Prinia }\end{array}$ & $\begin{array}{l}\text { Cisticoloa juncidis } \\
\text { Prinia flaviventris } \\
\text { Prinia inornata } \\
\text { Prinia socialis }\end{array}$ & $\begin{array}{l}\mathrm{R} \\
\mathrm{R} \\
\mathrm{R} \\
\mathrm{R}\end{array}$ & $\begin{array}{l}\text { Uncommon } \\
\text { Fairly common } \\
\text { Fairly common } \\
\text { Fairly common }\end{array}$ & $\begin{array}{l}\mathrm{B} \\
\mathrm{B} \\
\mathrm{B}\end{array}$ & $\begin{array}{l}\text { MF } \\
\text { MF } \\
\text { MF } \\
\text { MF }\end{array}$ & $\begin{array}{l}\text { AKN } \\
\text { GGV } \\
\text { GGV } \\
\text { GGV }\end{array}$ \\
\hline
\end{tabular}




\begin{tabular}{|c|c|c|c|c|c|c|}
\hline Common Name & Scientific Name & Status & Frequency & Breeding & Habitat & Observers \\
\hline $\begin{array}{l}\text { Paddy Field Warbler } \\
\text { Blyth's Reed-warbler } \\
\text { Indian Great Reed-warbler } \\
\text { Common Tailor Bird } \\
\text { Common Chiffchaff }\end{array}$ & $\begin{array}{l}\text { Acrocephalus agricola } \\
\text { Acrocephalus dumetorum } \\
\text { Acrocephalus stentoreus } \\
\text { Orthotomus sutorius } \\
\text { Phylloscopus collybita }\end{array}$ & $\begin{array}{l}\text { WM } \\
\text { WM } \\
\text { WM } \\
\text { R } \\
\text { WM }\end{array}$ & $\begin{array}{l}\text { Common } \\
\text { Uncommon } \\
\text { Uncommon } \\
\text { Common } \\
\text { Fairly Common }\end{array}$ & $\mathrm{B}$ & $\begin{array}{l}\text { MF } \\
\text { MF } \\
\text { MF } \\
\text { MF } \\
\text { MF }\end{array}$ & $\begin{array}{l}\text { AKN } \\
\text { AKN } \\
\text { GGV } \\
\text { BP/AKN/GGV } \\
\text { BP/AKN/GGV }\end{array}$ \\
\hline $\begin{array}{l}\text { Family: Musicapinae } \\
\text { Blue-throated Flycatcher } \\
\text { Red-throated Flycatcher } \\
\text { Verditer Flycatcher }\end{array}$ & $\begin{array}{l}\text { Cyornis rubecoloides } \\
\text { Ficedula parva } \\
\text { Eumyias thalassina }\end{array}$ & $\begin{array}{l}\text { WM } \\
\text { WM } \\
\text { WM }\end{array}$ & $\begin{array}{l}\text { Rare } \\
\text { Uncommon } \\
\text { Uncommon }\end{array}$ & & $\begin{array}{l}\text { MF } \\
\text { MF } \\
\text { MF }\end{array}$ & $\begin{array}{l}\text { AKN } \\
\text { AKN } \\
\text { BP }\end{array}$ \\
\hline $\begin{array}{l}\text { Family: Monarchinae } \\
\text { Asian Paradise-flycatcher } \\
\text { Black-naped Monarch-flycatcher }\end{array}$ & $\begin{array}{l}\text { Tarpsiphone paradisi } \\
\text { Hypothymis azurea }\end{array}$ & $\begin{array}{l}\mathrm{R} \\
\mathrm{R}\end{array}$ & $\begin{array}{l}\text { Uncommon } \\
\text { Uncommon }\end{array}$ & $\begin{array}{l}B \\
P B\end{array}$ & $\begin{array}{l}\text { MF } \\
\text { MF }\end{array}$ & $\begin{array}{l}\text { BP/AKN/GGV } \\
\text { BP/AKN/GGV }\end{array}$ \\
\hline $\begin{array}{l}\text { Family: Rhipidurinae } \\
\text { White-throated Fantail-flycatcher }\end{array}$ & Rhipidura albicollis & $\mathrm{R}$ & Fairly common & B & MF & BP/AKN/GGV \\
\hline $\begin{array}{l}\text { Family: Pachycephalinae } \\
\text { Mangrove Whistler }\end{array}$ & Pachycephala grisola & $\mathrm{R}$ & Rare & B & MF & $\mathrm{BP} / \mathrm{AKN}$ \\
\hline $\begin{array}{l}\text { Family: Paridae } \\
\text { Great Tit }\end{array}$ & Parus major & $\mathrm{R}$ & Common & $\mathrm{B}$ & MF & $\mathrm{BP} / \mathrm{AKN} / \mathrm{GGV}$ \\
\hline $\begin{array}{l}\text { Family: Nectariniidae } \\
\text { Purple-rumped Sunbird } \\
\text { Olive-backed Sunbird } \\
\text { Purple Sunbird }\end{array}$ & $\begin{array}{l}\text { Nectarina zeylonica } \\
\text { Nectarina jugularis } \\
\text { Nectarina asiatica }\end{array}$ & $\begin{array}{l}\mathrm{R} \\
\mathrm{WM} \\
\mathrm{R}\end{array}$ & $\begin{array}{l}\text { Fairly Common } \\
\text { Rare } \\
\text { Fairly Common }\end{array}$ & B & $\begin{array}{l}\text { MF } \\
\text { MF } \\
\text { MF }\end{array}$ & $\begin{array}{l}\text { BP/AKN/GGV } \\
\text { AKN } \\
\text { BP/AKN/GGV }\end{array}$ \\
\hline $\begin{array}{l}\text { Family: Zosteropidae } \\
\text { Oriental White-eye }\end{array}$ & Zosterops palpebrosus & $\mathrm{R}$ & Common & $\mathrm{B}$ & MF & $\mathrm{BP} / \mathrm{GGV}$ \\
\hline $\begin{array}{l}\text { Family: Estrildidae } \\
\text { White-throated Munia } \\
\text { Black-headed Munia } \\
\text { Spotted Munia }\end{array}$ & $\begin{array}{l}\text { Lonchura malabarica } \\
\text { Lonchura malacca } \\
\text { Lonchura punctulata }\end{array}$ & $\begin{array}{l}\mathrm{R} \\
\mathrm{R} \\
\mathrm{R}\end{array}$ & $\begin{array}{l}\text { Uncommon } \\
\text { Fairly Common } \\
\text { Uncommon }\end{array}$ & $\begin{array}{l}\mathrm{PB} \\
\mathrm{B}\end{array}$ & $\begin{array}{l}\text { MF/PF } \\
\text { MF } \\
\text { MF/PF }\end{array}$ & $\begin{array}{l}\text { GGV } \\
\text { BP/AKN/GGV } \\
\text { GGV }\end{array}$ \\
\hline $\begin{array}{l}\text { Family: Passerinae } \\
\text { House Sparrow }\end{array}$ & Passer domesticus & $\mathrm{R}$ & Uncommon & $\mathrm{B}$ & MF & GGV \\
\hline $\begin{array}{l}\text { Family: Ploceinae } \\
\text { Baya Weaver } \\
\text { Black-breasted Weaver }\end{array}$ & $\begin{array}{l}\text { Ploceus philippinus } \\
\text { Ploceus benghalensis }\end{array}$ & $\begin{array}{l}\mathrm{R} \\
\mathrm{R}\end{array}$ & $\begin{array}{l}\text { Common } \\
\text { Vagrant }\end{array}$ & $\begin{array}{l}\mathrm{B} \\
\mathrm{B}\end{array}$ & $\begin{array}{l}\text { MF } \\
\text { MF }\end{array}$ & $\begin{array}{l}\text { BP/AKN/GGV } \\
\text { GGV }\end{array}$ \\
\hline $\begin{array}{l}\text { Family: Sturnidae } \\
\text { Grey-headed Starling } \\
\text { Brahminy Starling } \\
\text { Asian Pied Starling } \\
\text { Common Myna } \\
\text { Jungle Myna }\end{array}$ & $\begin{array}{l}\text { Sturnus malabaricus } \\
\text { Sturnus Pagodarum } \\
\text { Sturnus contra } \\
\text { Acridotheres tristis } \\
\text { Acridotheres fuscus }\end{array}$ & $\begin{array}{l}\mathrm{R} \\
\mathrm{LM} \\
\mathrm{R} \\
\mathrm{R} \\
\mathrm{R}\end{array}$ & $\begin{array}{l}\text { Common } \\
\text { Rare } \\
\text { Common } \\
\text { Common } \\
\text { Common }\end{array}$ & $\begin{array}{l}\mathrm{B} \\
\mathrm{B} \\
\mathrm{B} \\
\mathrm{B}\end{array}$ & $\begin{array}{l}\text { MF } \\
\text { MF } \\
\text { MF } \\
\text { MF } \\
\text { MF }\end{array}$ & $\begin{array}{l}\text { BP/AKN/GGV } \\
\text { GGV } \\
\text { BP/AKN/GGV } \\
\text { BP/AKN/GGV } \\
\text { BP/AKN/GGV }\end{array}$ \\
\hline $\begin{array}{l}\text { Family: Oriolidae } \\
\text { Black-headed Oriole } \\
\text { Eurasian Golden Oriole }\end{array}$ & $\begin{array}{l}\text { Oriolus xanthornus } \\
\text { Oriolus oriolus }\end{array}$ & $\begin{array}{l}\mathrm{R} \\
\mathrm{R}\end{array}$ & $\begin{array}{l}\text { Common } \\
\text { Uncommon }\end{array}$ & $\mathrm{B}$ & $\begin{array}{l}\text { MF } \\
\text { MF }\end{array}$ & $\begin{array}{l}\text { BP/AKN/GGV } \\
\text { AKN/GGV }\end{array}$ \\
\hline $\begin{array}{l}\text { Family: Dicruridae } \\
\text { Black Drongo } \\
\text { Ashy Drongo } \\
\text { White-bellied Drongo }\end{array}$ & $\begin{array}{l}\text { Dicrurus macrocercus } \\
\text { Dicrurus leucophaeus } \\
\text { Dicrurus caerulescens }\end{array}$ & $\begin{array}{l}\mathrm{R} \\
\mathrm{R} \\
\mathrm{R}\end{array}$ & $\begin{array}{l}\text { Common } \\
\text { Common } \\
\text { Rare }\end{array}$ & $\mathrm{B}$ & $\begin{array}{l}\text { MF } \\
\text { MF } \\
\text { MF }\end{array}$ & $\begin{array}{l}\mathrm{BP} \\
\mathrm{AKN} / \mathrm{GGV} \\
\mathrm{BP} / \mathrm{AKN} / \mathrm{GGV}\end{array}$ \\
\hline $\begin{array}{l}\text { Family: Corvidae } \\
\text { Indian Treepie } \\
\text { Jungle Crow } \\
\text { House Crow }\end{array}$ & $\begin{array}{l}\text { Dendrocitta vagabunda } \\
\text { Corvus macrorhynchos } \\
\text { Corvus splendens }\end{array}$ & $\begin{array}{l}\mathrm{R} \\
\mathrm{R} \\
\mathrm{R}\end{array}$ & $\begin{array}{l}\text { Common } \\
\text { Common } \\
\text { Common }\end{array}$ & $\begin{array}{l}\mathrm{B} \\
\mathrm{B} \\
\mathrm{B}\end{array}$ & $\begin{array}{l}\text { MF } \\
\text { MF } \\
\text { MF }\end{array}$ & $\begin{array}{l}\text { BP/AKN/GGV } \\
\text { BP/AKN/GGV } \\
\text { BP/AKN/GGV }\end{array}$ \\
\hline
\end{tabular}

Abbreviations to observers initials: BP - Bivash Pandav; AKN - Anup Kumar Nayak; KA - Kalpana Ambastha; GGV - Gopi G V

Nomenclature follows Manakadan and Pittie (2002). The checklist was prepared while carrying out an ecological study on the colonial nesting waterbirds from August 2004 to December 2006.

fields and intertidal zones along the Gahirmatha Marine Sanctuary provide an ample habitat for these wintering species. These waders arrive during late October and depart around mid December with large concentrations of these birds are observed during mid November to second week of December. We speculate that this wetland is being used as a stop over site by the birds wintering in Chilika Lake.

\section{Additional data/confirmation required}

Certain species listed by Nayak (2003) including Plain Leaf Warbler, Brownish-flanked Bush Warbler, Garden Warbler, Ruddy Kingfisher, Grey-backed Shrike, Singing Bushlark, Whitethroated Bulbul, Thick-billed Green Pigeon, Saker Falcon, Pallid Scops Owl etc. need further confirmation for site record or require to be removed from the Bhitarkanika checklist. 


\section{Major conservation issues and implications}

Aquaculture farms: In the past few decades, aquaculture has experienced rapid growth in India and Orissa had been witnessing the same trend. Along the coastal belt of Orissa shrimp farming has been expanding vigorously. The expansion is driven by the high profitability of shrimp farming and attracts a wide range of investors, ranging from individual farmers (converting paddy fields) to multinational companies investing in large-scale semiintensive and intensive shrimp farming. This fast development of the shrimp sector is at the cost of the conversion of flat, agricultural and coastal lands to shrimp ponds. In December 1996, the Supreme Court responded by placing a number of stringent restrictions on shrimp farming in the coastal zone, including: No shrimp culture ponds were to be constructed within $500 \mathrm{~m}$ of the high tide mark, applicable for all seas, estuaries, creeks, rivers and backwaters; agricultural lands, salt pan lands, mangroves, wetland, forest lands and land for village common purposes were not to be converted into shrimp ponds.

However, this is being violated in this region and paddy fields are increasingly being converted to aquaculture farms. A total of 674 aquaculture farms (with total area of 793.45acres) were located along the peripheral region of the National Park alone. This illegal conversion of coastal wetlands will result in loss of foraging grounds and wintering grounds for a variety of bird species.

Poisoning and trapping of migratory birds: More than 90 species of birds visit Bhitarkanika with the onset of winter. Some of these birds are either trapped or poisoned by locals for consumption. Poisons are generally mixed in food grains and are kept on broad leaves for the birds to feed.

Conservation of Bhitarkanika birds is not only of local importance but also of global interest. In Bhitarkanika, we consider it important to intensify surveys of birds to obtain complete inventories of the avifauna. Further studies to determine the status and distribution of Indian skimmers, biology of sympatric kingfishers, identification of foraging corridors along with monitoring post dispersal movement patterns of colonial nesting waterbirds, listing of breeding birds and assesing Sathabaya wetland which has received little ornithological attention and no protection, could be taken up in near future to enrich the ornithological knowledge of this region. Finally, we stress the importance of documenting new or rare species records; photographs, video recordings and sound-recordings, as opposed to sight records, which can be objectively reviewed in the future and can therefore be considered as "reliable evidence".

\section{REFERENCES}

Chadah, S. \& C.S. Kar (1999). Bhitarkanika, Myth and Reality. Natraj Publisher, Dehradun, 388pp.

Chaudhari, A.B. \& Chakrabarti (1973). Wildlife biology of Sunderbans forests. A study of the birds of Sunderbans, with special reference to the breeding of openbill stork, little cormorant and large egret. Science Culture 39: 8-16.

Dani, C.S., S. Kar \& B.K. Behura (1999). Bhitarkanika - An unique Mangrove eco-system. Nature and Wildlife Conservation Society of Orissa. Bhubaneswar, India, pp. 30-43.

Gopi, G.V. \& B. Pandav (2007). Observations on breeding biology of three stork species in Bhitarkanika mangroves, India. Indian Birds 3(2): 45-50. Gopi, G.V., B. Pandav \& S.K. Kar (2005). An ecological reconnaissance of colonial nesting birds in Bhitarkanika mangroves, Orissa. Progress report
No.1. Unpublished report. Wildlife Institute of India, Dehradun, 28pp. Gopi, G.V., A.K. Jena \& B. Pandav (2006). Bhitarkanika Wildlife Sanctuary (Orissa), a key congregation area for Indian skimmer Rynchops albicollis. Birding Asia 5: 78.

Kalpana, A. (2005). Records of Red-winged Crested Cuckoo Clamator coromandus from Bhitarkanika National Park, Orissa. Indian Birds 1(5): 118. Manakadan, R. \& A. Pittie (2002). Standardised common and scientific names of the birds of the Indian subcontinent. Newsletter for Birdwatchers 42(3): i-viii, $1-36$

Mukerjee, A.K. (1959). Pakhiral, Sajnakhali - an introduction to a bird sanctuary in Sunderbans. Journal of Bengal Natural History Society 30: 161165.

Nitsure, S.R., M. Pejaver \& G. Quadros (2002). Species diversity of avifauna at Thane Creek near Rutuchakkra Nature Park, pp.276-282. In: Proceedings of the National Seminar on Creeks, Estuaries and Mangroves - Pollution and Conservation. Vidya Prasarak Mandal's B.N. Bandodkar College of Science, Thane (India).

Nayak, A. (2003a). Preliminary Field Guide to the Birds of Bhitarkanika. Mangrove Forest Division (Wildlife), Rajnagar, Orissa, 32pp.

Nayak, A. (2003b). Nesting ecology of resident birds in the Bhitarkanika Wildlife Sanctuary. Cheetal 41(3\&4): 43-54.

Nayak, A. (2005). Seasonal diversity of kingfishers in Bhitarkanika National Park, Orissa. Indian Forester 131(12): 1559-1563.

Pandav, B. (1997). Birds of Bhitarkanika mangroves, eastern India. Forktail 12: 7-17.

Prasad, S.N. \& K.P. Singh (1992). An ecological reconnaissance of Mangala in Krishna Estuary: Plea for conservation, pp.215-227. In: Singh, K.P. \& J.S. Singh (eds.). Tropical Ecosystems: Ecology $\mathbb{E}^{2}$ Management. Wiley Eastern Ltd., New Delhi, India.

Ripley, S.D. (1979). Changes in the bird fauna of a forest area; Simlipal hills, Mayurbhanj district and Dhenkanal district, Orissa. Journal of the Bombay Natural History Society 75(3): 570-574.

Samant, J.S. \& L.J. Bhosale (1986). Avifauna of the mangroves around Ratnagiri, Maharashtra, pp.456-466. In: Proceedings of the National Symposium on Biology, Utilization and Conservation of Mangroves. Shivaji University, Kolhapur, India.

Sethuraman, A., V.M. Karunagaran \& A.N. Subramanian (1997). Coastal wetlands of Pichavaram Mangrove forest, pp.22-23. In: Proceedings of the National Seminar on Coastal Zone Environment Management: An Appraisal of the Contemporary Research and Development. Karnataka, India.

Stanley, O.D. \& G. Quadros (2002). Biodiversity and ecology of the Gulf of Kachchh mangroves, Gujarat, pp.78-83. In: Proceedings of the National Seminar on Creeks, Estuaries and Mangroves - Pollution and Conservation. Vidya Prasarak Mandal's B.N. Bandodkar College of Science, Thane (India), 240pp.

Subramanya, S. (1996). Distribution, status and conservation of Indian heronries. Journal of the Bombay Natural History Society 93(3): 459-486.

Sen, S.K. Sunderbans Trip Report. Available on http:// www.kolkatabirds.com/sundertripreport.htm. Accessed on: 02.02.2006

Verma, A., N. Chaturvedi, S. Balachandran, I. Kehimkar \& G. Quadros (2002). Avian diversity in and around mangroves of Mahul Creek, Mumbai, India, pp.266-275. In: Proceedings of the National Seminar on Creeks, Estuaries and Mangroves - Pollution and Conservation. Vidya Prasarak Mandal's B.N. Bandodkar College of Science, Thane (India).

\section{ACKNowledgements}

The authors are grateful to The Director, Wildlife Institute of India for the facilities and infrastructure. The work is also benefited from the comments and advice from Mr. Rajah Jayapal (WII Dehradun) which largely improved the earlier draft. We wish to record our sincere thanks to the forest department staff at Bhitarkanika for providing necessary logistics and support. Our sincere thanks are due to Mr. Anup Nayak, IFS for his timely help in providing references. Lastly, we wish to acknowledge Mr. Bijoy Kumar Das and Mr. Kirodh Behera for their unfailing help during fieldwork.

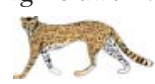

